\title{
AQUISIÇÃO DE VERBOS EM PRÉ-ESCOLARES FALANTES DO PORTUGUÊS BRASILEIRO
}

\section{Verb acquisition in Brazilian Portuguese speaking preschoolers}

\author{
Debora Maria Befi-Lopes ${ }^{(1)}$, Ana Manhani Cáceres ${ }^{(2)}$, Karina de Araújo ${ }^{(3)}$
}

\begin{abstract}
RESUMO
Objetivos: analisar quantitativamente a relação entre o uso de substantivos e verbos em situação de fala espontânea em pré-escolares em desenvolvimento normal de linguagem, bem como analisar a classificação dos verbos utilizados na mesma amostra de fala. Métodos: amostras de fala de sessenta pré-escolares (divididos em três grupos de acordo com sua faixa etária e pareados quanto ao sexo) obtidas em contexto educacional por meio de interação lúdica. Resultados: a análise dos dados demonstrou que o uso de verbos predominou em todos os grupos não havendo diferenciação entre os sexos. Com relação aos tipos de verbos, os sexos têm desempenho semelhante: o de maior ocorrência foi o intransitivo, sendo que em Gl e Gll o segundo mais freqüente foi o de ligação e em GIII foi o transitivo direto. Conclusão: este estudo demonstrou que os pré-escolares estudados utilizam mais verbos que substantivos desde o segundo ano de vida, sendo que o verbo mais freqüente foi de tipo intransitivo em todas as faixas etárias seguido pelo verbo de ligação nos segundo e terceiro anos de vida e pelo transitivo direto no quarto ano de vida. Finalmente, constatou-se não haver diferenças significantes nos aspectos aqui mencionados para meninas e meninos.
\end{abstract}

DESCRITORES: Desenvolvimento da Linguagem; Linguagem Infantil; Vocabulário; Criança

\section{INTRODUÇÃO}

O processo de aquisição da linguagem inicia-se desde os primeiros minutos da vida de um bebê, pois já em seu nascimento estruturas responsáveis pelo processamento da linguagem estão em desenvolvimento. O reforço para este processo decorrerá de experiências vividas, relações sociais e interações comunicativas. Em outras palavras,

(1) Fonoaudióloga; Professora Associada do Departamento de Fisioterapia, Fonoaudiologia e Terapia Ocupacional da Faculdade de Medicina da Universidade de São Paulo; Livre-Docente do Curso de Fonoaudiologia da Faculdade de Medicina da Universidade de São Paulo.

(2) Estudante de Fonoaudiologia do Departamento de Fisioterapia, Fonoaudiologia e Terapia Ocupacional da Faculdade de Medicina da Universidade de São Paulo.

(3) Fonoaudióloga; Colaboradora do Laboratório de Investigação Fonoaudiológica em Desenvolvimento da Linguagem e suas Alterações do Curso de Fonoaudiologia da Faculdade de Medicina da Universidade de São Paulo; Doutora em Semiótica e Lingüística Geral pela Faculdade de Filosofia, Letras e Ciências Humanas da Universidade de São Paulo.

Artigo baseado nos resultados do projeto de Iniciação Científica financiado pela Fundação de Amparo à Pesquisa do Estado de São Paulo - FAPESP. é possível perceber que o neurodesenvolvimento está condicionado a aspectos biológicos, sociais e ambientais ${ }^{1}$.

$O$ aspecto social da linguagem permite que vocabulário e cognição se desenvolvam conjuntamente a partir de interações sociais ${ }^{2}$. A criança aprende como sua língua categoriza o mundo para então poder conhecer o significado da fala dos adultos ${ }^{3}$. Assim, compreendendo a linguagem como primeira forma de socialização, o papel exercido pelo input lingüístico é enfatizado ${ }^{4}$. As principais características da fala direcionada à criança (FDC) são a simplificação sintática, o vocabulário delimitado, a redução da complexidade proposicional e fluência mais adequada ${ }^{5}$. Esta fala - que é especialmente utilizada pela mãe - pode ser compreendida como uma resposta às necessidades do bebê, permitindo que este se insira numa relação de diálogo. Entretanto, deve estar ligeiramente além de sua capacidade a fim de se consistir num estímulo para seu avanço lingüístico.

Em contrapartida, alguns autores buscando comprovar que a aquisição da linguagem baseia-se em estruturas inatas, realizaram um estudo sobre a aquisição sintática e, a partir de seus resultados, concluíram que desde o início da combinação de 
palavras as crianças já possuem uma rica estrutura sintática - incompatível com a possibilidade de ser decorrente da exposição à língua ${ }^{6}$. Assim, supõem haver na mente representações que guiam a estruturação dos estímulos de forma a tornar a aquisição possível. Já o modelo de uso-baseado ${ }^{7,8}$ - atentando para o aspecto cultural da linguagem - propõe que a criança aprenda expressões lingüísticas concretas a partir da imitação do que escuta; em outros termos, a linguagem é adquirida através do uso. Logo, é utilizando suas habilidades cognitivas e sociais que ela categoriza, esquematiza e combina, de forma criativa, as expressões que aprendeu separadamente.

Observando o desenvolvimento típico da linguagem, pode-se verificar que em seu primeiro aniversário a criança deve ter proferido sua primeira palavra - que provavelmente possuía um referente bastante familiar e era utilizada genericamente para nomear objetos com alguma semelhança em situações diversas ${ }^{9}$. Será próximo ao seu segundo aniversário que ela deverá vivenciar a chamada explosão do vocabulário - fase em que aprende novas palavras com facilidade e rapidez surpreendentes - aumentando significativamente seu vocabulário.

Ao analisar o desenvolvimento gramatical das crianças e a ampliação de seu vocabulário é necessário considerar que substantivos e verbos possuem características distintas. De forma bem sucinta, o substantivo geralmente corresponde ao nome que se dá a algo (ou alguém) e freqüentemente funciona como argumento, ao contrário do verbo que costuma expressar ações e processos e ter como característica funcionar como predicado ${ }^{10}$. Os verbos possuem grande variedade semântica $e$ gramatical o que impede sua rápida generalização; logo, as crianças precisam ser expostas muitas vezes ao mesmo verbo para aprenderem suas propriedades ${ }^{11}$. Ele consiste, portanto, numa palavra cujo referente não é tão claro quanto o do substantivo; assim, sua aquisição costuma ocorrer de forma mais gradual.

Contextos ostensivos - aqueles nos quais, palavra e referente são apresentados no campo visual da criança simultaneamente - não são favoráveis para crianças pequenas aprenderem verbos; pois ela provavelmente encontra dificuldades em determinar o foco da atenção do adulto, afinal, este costuma utilizar verbos para antecipar ou direcionar seu comportamento - situações nas quais o referente não está presente durante sua enunciação ${ }^{12}$. A aquisição dos verbos sofre influências também de sua freqüência nos estímulos (input): quanto maior for a freqüência de um verbo no estímulo, mais e com maior flexibilidade ele aparecerá na fala da criança ${ }^{13}$. Vale salientar que as proprieda- des englobadas pela freqüência de verbos nos estímulos são: freqüência total, freqüência em posição final e diversidade de contextos sintáticos em que aparecem.

Um outro fator que parece favorecer a escassez de verbos na fala - em seu período inicial - consiste no privilégio que a fala materna tende a dar aos substantivos ao invés dos verbos. Em outras palavras, pelo fato de os verbos estarem mais relacionados a comportamentos não verbais do que a comportamentos verbais, os pais influenciam seus filhos a nomear objetos muito mais do que nomear ações ${ }^{14}$. Tais evidências encaminham ao entendimento de que esta discrepância verificada no princípio da produção entre substantivos e verbos é resultado da interação da criança com a linguagem - intermediada pelo adulto - que estimula a compreensão dos verbos, mas não a incentiva a produzi-los.

O uso de verbos favorece o desenvolvimento gramatical, pois determina a superação dos enunciados de uma palavra para as combinações de diversas palavras, assim entre o terceiro e o quarto ano de vida seu uso é aperfeiçoado ${ }^{8,15}$. Tal aquisição - comparada a dos substantivos - demonstra que quando a criança possui entre cinqüenta e cem palavras seu inventário de verbos começa a aumentar, estendendo-se até o momento em que ela possui cerca de quinhentas palavras em seu vocabulário; a partir deste instante ocorre a equiparação entre a proporção de verbos e demais palavras ${ }^{16}$.

Isto pode ser observado num estudo com crianças chinesas - que foram adotadas e aprenderam o inglês como segunda língua - onde inicialmente os substantivos eram mais facilmente aprendidos que os verbos, e com a ampliação do vocabulário, os verbos foram aumentando até se equipararem ${ }^{17}$. Em um estudo de caso de uma criança de 20 meses falante do Português Brasileiro constatou-se que a diferença entre a produção de substantivos e verbos é pequena, além de a explosão do vocabulário englobar ambas as categorias gramaticais ${ }^{18}$.

Mesmo dentro da classe dos verbos observamse particularidades de aquisição. Os verbos de movimento são favorecidos pela condição futura - verbo enunciado antes do evento -, ao passo que, os de resultado são favorecidos pela condição completa - verbo enunciado após o evento ${ }^{19}$. Estes dados sugerem que o momento de apresentação do verbo age como uma pista para direcionar o foco do evento ao qual este se refere. A organização semântica destes Ihes confere distinções, uma vez que suas características determinam como serão armazenados. Sendo assim, quanto mais um item compartilhar elementos com outros mais facilmente 
ele será armazenado e acessado. Assim, verbos concretos geram códigos de memória mais fortes, sendo acessados mais facilmente do que verbos simples ${ }^{20}$.

Outra diferença relatada diz respeito ao tipo de referência do verbo, pois aqueles que se referem a ações físicas (por exemplo, puxar) são identificados com maior freqüência do que aqueles que se referem a estados mentais (por exemplo, querer) ${ }^{21}$. Pode-se verificar a habilidade de generalizar verbos familiares para novos eventos por meio do vocabulário expressivo da criança. Assim, partindo do pressuposto de que este vocabulário representa uma medida de sua aquisição lexical, pode-se afirmar que quanto mais a criança vivencia a linguagem melhor ela compreende os princípios do aprendizado verbal ${ }^{22}$.

Ao buscar a compreensão de como as crianças apreendem os significados das palavras, especialmente dos verbos, é possível encontrar duas teorias. A primeira é a teoria das "Amarras Semânticas", fortemente baseada na observação do mundo, que prevê que o aprendizado da gramática ocorra pelas relações entre objetos concretos com a categoria gramatical dos substantivos, e entre ações com a categoria dos verbos. A segunda é a teoria das "Amarras Sintáticas", prevê que a utilização de contextos sintáticos, nos quais o verbo está inserido para a suposição de seu significado. De acordo com esta última, a criança é sensível às relações entre os substantivos e os argumentos, sendo capaz de identificar o verbo (ou o predicado) através de um processo de eliminação. Isto significa dizer que elas deduzem o significado do verbo a partir da contribuição fornecida pelo contexto sintático somada à observação do contexto extralingüístico ${ }^{23,24}$.

Com base em quatro experimentos realizados com crianças entre 18 e 30 meses, buscando verificar se estas eram capazes de utilizar argumentos verbais para obter informações sobre o significado do verbo, os autores concluíram que as crianças podem usar a estrutura sintática para aprender o significado de verbos novos ${ }^{23}$.

A descoberta da relação entre a morfologia e os conceitos de tempo verbal e aspecto permite que a criança construa representações a respeito da estrutura do predicado. Assim, se ela pode pensar sobre eventos remotos no tempo e no espaço, possui uma base conceitual que lhe fornece elementos para a construção de um sistema lingüístico; este, por sua vez, a permite falar sobre relações temporais. Portanto, a interface semântica-sintaxe é um componente fundamental para a aquisição da linguagem ${ }^{25,26}$. A compreensão do significado dos verbos estaria relacionada às diferentes interações que uma ação pode determinar sobre um objeto.
Assim, supõe-se que a informação estrutural aliada à informação contextual - seja fundamental para a fixação do significado de verbos novos pela criança ${ }^{27}$.

A classificação dos verbos de acordo com a tipologia dos eventos por eles expressos parece, portanto, ser pertinente tanto para a compreensão das peculiaridades de comportamento sintático e semântico destes na frase, quanto na compreensão do papel que desempenham no aprendizado infantil ${ }^{28}$.

Com relação ao argumento verbal, verificou-se que em crianças falantes do francês sua aquisição corresponde a um longo processo que está sujeito a mecanismos lexicais que ainda não estão dominados no quinto ano de vida. A assimetria deste processo quanto ao contexto sintático também fica evidente, pois, verbos transitivos recusam contextos intransitivos antes de verbos intransitivos recusarem contextos transitivos ${ }^{29}$. Quando comparadas crianças chinesas e brasileiras constatou-se que a aquisição dos verbos ocorre antes para as chinesas. Entretanto, verificou-se também que os adultos chineses utilizam verbos mais específicos do que os brasileiros, pois os verbos na língua chinesa carregam mais informações sobre as ações, sendo mais concretos e aplicáveis em contextos restritos. Tal fato parece torná-los mais salientes, fornecendo pistas que contribuem para sua memorização ${ }^{3}$.

Com relação ao Português Brasileiro, sabese que é entre dois anos e dois anos e meio que as crianças geralmente começam a utilizar - com certa propriedade - os verbos em seus enunciados ${ }^{30}$. A interação com o meio lingüístico favorece seu aperfeiçoamento e esta, além de aumentar o uso dos verbos, passa a utilizá-los para estruturar suas frases respeitando a ordem temporal dos acontecimentos.

Vale ressaltar que os verbos do Português Brasileiro podem ser agrupados em verbos significativos e verbos designativos (ou não significativos ou de ligação). Os verbos considerados designativos são apenas ser, estar, continuar, permanecer e ficar ${ }^{31}$. Os demais podem ser classificados de acordo com seu complemento em intransitivos, transitivos indiretos, transitivos diretos, transitivos relativos, transitivos circunstanciais, bitransitivos, transobjetivos e de ligação ${ }^{32}$.

A partir do exposto na literatura e considerando a importância que a utilização correta de verbos tem para a eficácia de comunicação em crianças, o presente estudo tem como objetivos: (1) demonstrar quantitativamente a relação entre o uso de substantivos e verbos em situação de fala espontânea em pré-escolares em desenvolvimento normal de linguagem e (2) analisar a classificação dos verbos utilizados na mesma amostra de fala. 


\section{MÉTODOS}

Todos os sujeitos estavam matriculados em creches do município de São Paulo, localizadas na zona norte da referida cidade. As instituições mantinham convênio com a prefeitura e atendiam, na maioria, crianças de classe econômica média-baixa e baixa. Com o objetivo de caracterizar o desempenho de linguagem destas foi realizada uma triagem fonoaudiológica ${ }^{33}$ que verificava as habilidades de vocabulário, fonologia, produção e compreensão de sentenças. Após a realização da triagem, foram selecionadas sessenta crianças, em idade pré-escolar, com desenvolvimento normal de linguagem.

Previamente à coleta de dados, os pais foram esclarecidos a respeito dos objetivos da pesquisa e da forma de obtenção dos dados para somente então assinarem o Termo de Consentimento Livre e Esclarecido.

Os sujeitos foram divididos em três grupos conforme a faixa etária a qual pertenciam, observando que cada grupo deveria conter vinte sujeitos, sendo dez do sexo feminino e dez do masculino. O Grupo 1 (Gl) comporta a faixa etária de 2:0 a 2:11 anos, com média de 2 anos e 5 meses; o Grupo 2 (GII) comporta a faixa etária de 3:0 a 3:11 anos, com média de 3 anos e 4 meses; e o Grupo 3 (GIII) comporta a faixa etária de 4:0 a 4:11 anos, com média de 4 anos e 4 meses.

Considerando as faixas etárias estudadas e a necessidade de obtenção de fala espontânea, os dados (amostra de fala) foram coletados a partir de situação de interação lúdica com brinquedos, a saber: miniatura de fazenda com animais, meios de transporte, alimentos, utensílios de cozinha e dois bonecos.

Além dos brinquedos, foram utilizados uma filmadora VHS, fitas de vídeo (uma para cada sujeito), um gravador e fitas cassete (também uma para cada sujeito).

Para a coleta dos dados, a pesquisadora preparou a sala concedida para tal fim, posicionando a filmadora sobre um tripé e o gravador cassete ao lado da criança e dispondo os brinquedos sobre um tapete. Todos os brinquedos foram dispostos da mesma maneira para todos os sujeitos sendo que os utensílios de cozinha ficavam localizados à frente e no centro, os animais e fazendinha à direita e os meios de transporte e bonecos à esquerda.

Cada uma das crianças era solicitada a se retirar de sua sala de aula e, então, era levada à sala previamente preparada. A criança era encorajada a interagir com a pesquisadora por um período de trinta minutos. Durante a interação, a pesquisadora realizou perguntas abertas com o objetivo de propiciar a melhor situação de iniciativa por parte da criança. Além disso, reduziu as perguntas fechadas que necessitassem de respostas pontuais como sim ou não. Deve-se ressaltar que os brinquedos utilizados propiciaram uma situação de comunicação efetiva, propiciando comentários e relatos por parte das crianças, inclusive as mais novas. Cada situação foi filmada em VHS e gravada em cassete.

Após a coleta dos dados, foi realizada a transcrição das amostras de fala. Para cada transcrição, o mesmo procedimento foi adotado: primeiramente, a pesquisadora assistia toda a gravação sem realizar nenhuma anotação, para observar o desempenho geral da criança. Num segundo momento, ela assistia a gravação realizando a transcrição. A amostra de fala foi dividida em seguimentos (cada enunciado produzido pela criança), até que se chegasse ao número esperado de 100 segmentos ${ }^{34}$. Quando este número foi atingido, o restante da transcrição foi desprezado não fazendo parte da análise. Para que se pudesse perceber e dividir corretamente cada segmento pelo menos um dos critérios deveria ocorrer: mudança de assunto por parte da criança, mudança do foco de atenção da criança, ou interrupção do terapeuta. Outro critério adotado na transcrição foi a utilização de segmentos completamente transcritos, nenhum com quebras. Todas as repetições exatas de segmentos foram incluídas. As disfluências, como as repetições de palavra, foram pontuadas uma única vez. Em poucos casos, quando as palavras foram produzidas para dar ênfase, contou-se cada ocorrência.

Após a transcrição de todas as fitas, a pesquisadora procedeu ao levantamento dos dados, listando os substantivos e verbos para análise do primeiro objetivo do presente estudo. A seguir, procedeu à classificação dos verbos de acordo com o complemento expresso ${ }^{32}$ para análise do segundo objetivo desta pesquisa.

Vale ressaltar que a classificação dos verbos baseou-se no complemento por eles apresentado. Assim, cada verbo era analisado com relação ao complemento que apresentava no segmento em questão. Desta forma, um mesmo verbo poderia ser classificado de diferentes formas de acordo com o contexto em que fora emitido.

Este estudo foi autorizado pela Comissão de Ética para Análise de Projetos de Pesquisa da Diretoria Clínica do Hospital das Clínicas da Faculdade de Medicina da Universidade de São Paulo sob número 360/01.

Para a análise estatística dos resultados foram utilizados os seguintes testes: teste-t pareado e independente e a análise de variância (ANOVA) para as comparações entre os sexos e os grupos para cada uma das variáveis, supondo-se igualdade de variância e distribuição normal. Para as múltiplas 
comparações foi utilizado o teste de Tukey. O nível de significância adotado foi de $5 \%$. Os resultados significantes foram assinalados com um asterisco.

\section{RESULTADOS}

Os dados obtidos em cada grupo com relação à distribuição de substantivos e verbos podem ser comparados entre os grupos e também por sexo na Tabela 1. A comparação entre os verbos pode

Tabela 1 - Distribuição de substantivos e verbos por grupo

\begin{tabular}{llcc}
\hline Grupo & Sexo & Substantivo & Verbo \\
\hline \multirow{2}{*}{ GI } & Feminino & 525 & 681 \\
& Masculino & 507 & 712 \\
& Geral & $\mathbf{1 0 3 2}$ & $\mathbf{1 3 9 3}$ \\
\hline \multirow{3}{*}{ Gll } & Feminino & 520 & 831 \\
& Masculino & 545 & 754 \\
& Geral & $\mathbf{1 0 6 5}$ & $\mathbf{1 5 8 5}$ \\
\hline \multirow{2}{*}{ GIII } & Feminino & 660 & 991 \\
& Masculino & 583 & 931 \\
& Geral & $\mathbf{1 2 4 3}$ & $\mathbf{1 9 2 2}$ \\
\hline
\end{tabular}

Legenda: GI - grupo 1; GII - grupo 2; GIII - grupo 3. ser verificada na Tabela 2 tanto entre os grupos, quanto por sexo. A Tabela 3 mostra os resultados das comparações entre substantivos e verbos para cada grupo, onde se observa que em todos predomina significativamente o uso de verbos.

De acordo com os resultados do teste-t independente, os sexos não se diferenciam significativamente com relação à produção tanto de verbos, quanto de substantivos em nenhum dos grupos ou de forma geral (independente do grupo). Na Tabela 4 observa-se a comparação entre a ocorrência de substantivos e verbos para cada grupo e geral considerando-se apenas os sujeitos do sexo feminino e na tabela 5 apenas os do sexo masculino.

A partir das Tabelas 5 e 6 observa-se que apenas para GI não há diferença estatisticamente significativa entre o uso de substantivos e de verbos para ambos os sexos, apesar do p-valor ser muito próximo do nível de significância. Para as demais comparações o uso de verbos é sempre significativamente maior tanto para o sexo feminino quanto para o sexo masculino. Pode-se observar que em todos os grupos o tipo de verbo de maior ocorrência é o intransitivo. Em Gl e Gll o segundo mais freqüente é o de ligação e em GIII é o transitivo direto.

Tabela 2 - Distribuição dos verbos de acordo com sua classificação por grupo

\begin{tabular}{|c|c|c|c|c|c|c|c|c|c|c|}
\hline \multirow[b]{2}{*}{ Grupo } & \multirow[b]{2}{*}{ Sexo } & \multicolumn{8}{|c|}{ Verbo } & \multirow[b]{2}{*}{ Total } \\
\hline & & Intransitivo & $\begin{array}{l}\text { Transitivo } \\
\text { Direto }\end{array}$ & $\begin{array}{l}\text { Transitivo } \\
\text { Indireto }\end{array}$ & $\begin{array}{c}\text { Transitivo } \\
\text { Relativo }\end{array}$ & $\begin{array}{c}\text { Transitivo } \\
\text { Circuns- } \\
\text { tancial }\end{array}$ & Bitransitivo & $\begin{array}{l}\text { Transobje- } \\
\quad \text { tivo }\end{array}$ & de Ligação & \\
\hline & Feminino & 240 & 95 & 7 & 6 & 113 & 13 & 1 & 206 & 681 \\
\hline \multirow[t]{3}{*}{ GI } & Masculino & 306 & 115 & 11 & 0 & 122 & 5 & 0 & 153 & 712 \\
\hline & Geral & 546 & 210 & 18 & 6 & 235 & 18 & 1 & 359 & 1393 \\
\hline & Feminino & 295 & 218 & 27 & 5 & 69 & 9 & 3 & 205 & 831 \\
\hline \multirow[t]{3}{*}{ GII } & Masculino & 278 & 172 & 10 & 11 & 61 & 2 & 4 & 216 & 754 \\
\hline & Geral & 573 & 390 & 37 & 16 & 130 & 11 & 7 & 421 & 1585 \\
\hline & Feminino & 347 & 287 & 54 & 19 & 54 & 3 & 8 & 219 & 991 \\
\hline \multirow[t]{2}{*}{ GIII } & Masculino & 340 & 301 & 32 & 16 & 80 & 2 & 0 & 160 & 931 \\
\hline & Geral & 687 & 588 & 86 & 35 & 134 & 5 & 8 & 379 & 1922 \\
\hline
\end{tabular}

Legenda: Gl - grupo 1; GII - grupo 2; GIII - grupo 3.

Tabela 3 - Comparação entre substantivos e verbos em cada grupo

\begin{tabular}{llcc}
\hline \multicolumn{1}{r}{ Grupo } & t & p-valor \\
\hline GI & $\begin{array}{l}\text { Substantivo } \\
\text { Verbo }\end{array}$ & $-3,08$ & $\mathbf{0 , 0 0 6}^{*}$ \\
\hline Gll & $\begin{array}{l}\text { Substantivo } \\
\text { Verbo }\end{array}$ & $-5,76$ & $<\mathbf{0 , 0 0 1 ^ { * }}$ \\
\hline GIII & $\begin{array}{l}\text { Substantivo } \\
\text { Verbo }\end{array}$ & $-6,91$ & $<\mathbf{0 , 0 0 1 ^ { * }}$ \\
\hline
\end{tabular}

Legenda: GI - grupo 1; Gll - grupo 2; GIII - grupo 3.

Baseada no resultado do teste-t pareado.
Tabela 4 - Comparação entre substantivos e verbos em cada grupo e geral considerando apenas o sexo feminino

\begin{tabular}{llcc}
\hline \multicolumn{1}{c}{ Grupo } & \multicolumn{1}{c}{ t } & p-valor \\
\hline GI & $\begin{array}{l}\text { Substantivo } \\
\text { Verbo }\end{array}$ & $-2,00$ & 0,076 \\
\hline GII & $\begin{array}{l}\text { Substantivo } \\
\text { Verbo }\end{array}$ & $-5,16$ & $\mathbf{0 , 0 0 1 ^ { * }}$ \\
\hline GIII & $\begin{array}{l}\text { Substantivo } \\
\text { Verbo }\end{array}$ & $-5,74$ & $<\mathbf{0 , 0 0 1 ^ { * }}$ \\
\hline Geral & $\begin{array}{l}\text { Substantivo } \\
\text { Verbo }\end{array}$ & $-7,16$ & $<\mathbf{0 , 0 0 1 ^ { * }}$ \\
\hline
\end{tabular}

Legenda: GI - grupo 1; GII - grupo 2; GIII - grupo 3.

Baseada no resultado do teste-t pareado. 
Tabela 5 - Comparação entre substantivos e verbos em cada grupo e geral considerando apenas o sexo masculino

\begin{tabular}{llll}
\hline Grupo & & $\mathbf{t}$ & p-valor \\
\hline GI & $\begin{array}{l}\text { Substantivo } \\
\text { Verbo }\end{array}$ & $-2,25$ & $\mathbf{0 , 0 5 1}$ \\
\hline GII & $\begin{array}{l}\text { Substantivo } \\
\text { Verbo }\end{array}$ & $-3,16$ & $\mathbf{0 , 0 1 2 ^ { * }}$ \\
\hline GIII & $\begin{array}{l}\text { Substantivo } \\
\text { Verbo }\end{array}$ & $-4,21$ & $\mathbf{0 , 0 0 2 ^ { * }}$ \\
\hline Geral & $\begin{array}{l}\text { Substantivo } \\
\text { Verbo }\end{array}$ & $-5,45$ & $<\mathbf{0 , 0 0 1 ^ { * }}$ \\
\hline
\end{tabular}

Legenda: Gl - grupo 1; Gll - grupo 2; GIII - grupo 3.

Baseada no resultado do teste-t pareado.

Tabela 6 - Comparação entre os tipos de verbo em cada grupo

\begin{tabular}{lcl}
\hline & $\mathbf{f}$ & p-valor \\
\hline GI & 65,2 & $<\mathbf{0 , 0 0 1 ^ { * }}$ \\
GII & 114,13 & $<\mathbf{0 , 0 0 1 ^ { * }}$ \\
GIII & 130 & $<\mathbf{0 , 0 0 1 ^ { * }}$ \\
\hline
\end{tabular}

Legenda: GI - grupo 1; Gll - grupo 2; GIII - grupo 3. Baseada no resultado da ANOVA.

A Tabela 6 mostra os resultados da ANOVA para a comparação entre os tipos de verbo em cada grupo. Os resultados da ANOVA apontam que todos os grupos se diferenciam quanto aos tipos de verbo. De acordo com o teste de Tukey, em GI predomina significativamente o verbo intransitivo, seguido do verbo de ligação e dos transitivos direto e circunstancial, sendo que o uso dos dois últimos é igual. Os demais tipos de verbo apresentam ocorrência igual entre si e menor que os quatro citados anteriormente.

Para GII, o teste de Tukey aponta que o verbo intransitivo também predomina significativamente, seguido do verbo de ligação, transitivo direto, que são iguais entre si, e transitivo circunstancial. Os demais tipos de verbo apresentam ocorrência igual entre si e menor que os quatro citados anteriormente.

Em GIII, o teste de Tukey aponta que para GIII predomina significativamente verbo intransitivo, seguido do transitivo direto, de ligação e dos transitivos circunstancial e indireto, sendo que o uso dos dois últimos é igual. Os demais tipos de verbo apresentam ocorrência igual entre si e menor que os quatro citados anteriormente.

A Tabela 7 mostra os resultados da ANOVA para a comparação entre os tipos de verbo em sexo. Os resultados da ANOVA apontam que ambos os sexos se diferenciam quanto aos tipos de verbo. $O$ teste de Tukey aponta que para ambos os sexos
Tabela 7 - Comparação entre os tipos de verbo em cada sexo

\begin{tabular}{lcl}
\hline & $\mathbf{f}$ & p-valor \\
\hline $\mathrm{F}$ & 98,9 & $<\mathbf{0 , 0 0 1 ^ { * }}$ \\
$\mathrm{M}$ & 115,83 & $<\mathbf{0 , 0 0 1 ^ { * }}$ \\
\hline
\end{tabular}

Legenda: GI - grupo 1; GII - grupo 2; GIII - grupo 3.

Baseada no resultado da ANOVA.

predominam o verbo intransitivo, seguido do verbo transitivo direto e do de ligação que se diferenciam entre si. Na seqüência vem o transitivo circunstancial, seguido do transitivo indireto. Desta forma, o comportamento dos sexos é semelhante quanto à ocorrência dos tipos de verbo.

\section{DISCUSSÃO}

Combinando as informações verificadas na literatura com a análise estatística dos achados apresentam-se os resultados desta pesquisa. O primeiro objetivo compreendia a verificação quantitativa da relação entre o uso de substantivos e verbos em fala espontânea de pré-escolares. A observação da Tabela 3 evidencia que o uso de verbos foi significativamente maior que o de substantivos em todos os grupos.

Estes achados parecem divergir de relatos na literatura que apontam os substantivos como mais facilmente adquiridos devido a sua referência concreta $9,10,16,17$, enquanto os verbos possuem características que os tornam mais complexos, como sua referência mais abstrata $8,11,14,15,22,27,30$. Porém, uma verificação mais minuciosa pode sugerir que os sujeitos analisados - por estarem além do segundo ano de vida - já possuem o vocabulário em expansão ${ }^{16,22}$. Isto poderia ser fortalecido pela observação de um estudo de caso ${ }^{18}$ de que aos 20 meses a diferença entre substantivos e verbos é pequena no Português Brasileiro.

No Gl esta predominância dos verbos parece ser equivalente à explosão do vocabulário ${ }^{2,9}$, pois esta engloba tanto substantivos quanto verbos ${ }^{18}$. Nos Gll e GIII pode-se supor que este fato se deva ao aperfeiçoamento do uso dos verbos ${ }^{8,30}$. O estudo comparativo das línguas chinesa e portuguesa ${ }^{3}$ demonstra que apesar de crianças chinesas adquirirem verbos antes das brasileiras, o uso convencional dos verbos é equivalente entre estas populações.

Nota-se ainda que a média de ocorrência de verbos é maior em ambos os sexos em todos os grupos. Entretanto, não há diferenças significativas entre os sexos quanto ao uso de substantivos e verbos. 
A comparação destes achados no sexo femininoem cada um dos grupos e em geral - evidencia que apenas no GI não há diferença significativa entre o uso de verbos e substantivos. Nos demais casos, os verbos são significativamente mais freqüentes. O mesmo pode ser verificado para o sexo masculino, enfatizando não haver distinção entre os sexos nesta questão (Tabelas 4 e 5). Os achados relacionados ao sexo e a constatação de que não há diferenças significativas entre eles parece fortalecer tanto a importância de um aparato biológico adequado, quanto de um ambiente comunicativo saudável ${ }^{1-8}$.

Apesar de não haver ainda estudos que descrevam a classificação do uso dos verbos do Português Brasileiro, sabe-se que existem diferenças na aquisição dos verbos ${ }^{13,19-21,24,26}$. São apresentados aqui os resultados concernentes à análise dos verbos utilizados pelos pré-escolares.

O uso do verbo intransitivo foi o mais freqüente em todos os grupos, sendo importante destacar que muitos verbos apesar de não serem usualmente considerados como verbos intransitivos, eram assim utilizados de forma compreensível dentro de seu contexto de enunciação, como proposto neste estudo ${ }^{32}$.

Pela Tabela 6 verifica-se que todos os grupos se diferenciam quanto aos tipos de verbos. Os achados demonstram que em Gl o verbo intransitivo é seguido pelo de ligação e depois pelos transitivos direto e circunstancial; em Gll o verbo intransitivo é seguido pelos de ligação e transitivo direto e depois pelo transitivo circunstancial; em GIII o verbo intransitivo é seguido pelo transitivo direto, depois pelo transitivo circunstancial e, finalmente, pelos transitivos circunstancial e indireto.

Quando, na Tabela 7, se comparam os tipos de verbos com relação aos sexos, percebe-se que há diferenças, pois em ambos os sexos os verbos predominantes são o verbo intransitivo, o transitivo direto, o de ligação, o transitivo circunstancial e o transitivo indireto, respectivamente.

Esta evolução no uso dos verbos do Português Brasileiro nos parece compatível com os estudos que afirmam ser a aquisição dos verbos baseada no uso ${ }^{7,8}$; e na atenção às pistas fornecidas pelo contexto e pelas estruturas sintáticas e semânticas ${ }^{12-14,17,19,21,24,25,29}$. As análises e reflexões procedi- das neste artigo proporcionam informações essenciais para a compreensão do desenvolvimento típico da linguagem, não apenas no que se restringe aos aspectos gramaticais, mas que se ampliam para o contexto lingüístico dos pré-escolares.

\section{CONCLUSÃO}

Conclui-se que nesta amostra que na comparação entre substantivos e verbos, estes predominaram demonstrando sua importância desde períodos iniciais da aquisição gramatical. Considerando as particularidades apresentadas pelos verbos, o estudo buscou contribuir para a compreensão de seu processo de aquisição e evolução.

Foram constatadas diferenças entre a ocorrência dos tipos de verbos nos grupos considerados, sendo que o tipo de verbo mais freqüente em todos os grupos foi o intransitivo, sendo seguido pelos de ligação (GI e GII) e transitivo direto (GIII). Quanto aos sexos houve compatibilidade entre os resultados gerais, o que viabiliza concluir não haver diferenças significantes nos aspectos aqui mencionados para meninas e meninos.

A pesquisa indicou que as relações estudadas são pertinentes à compreensão do processo global de desenvolvimento da linguagem. Considerando a importância de acrescentar informações aos primeiros achados, em continuidade ao presente estudo, tais pesquisadoras estão realizando a verificação de tempo, modo, número e pessoa dos verbos classificados, bem como a verificação dos verbos produzidos com o intuito de evidenciar aqueles que são mais recorrentes e sua evolução ao longo das faixas etárias analisadas; tais dados poderão fornecer informações ainda mais consistentes favorecendo o avanço na compreensão da aquisição dos verbos do Português Brasileiro.

\section{AGRADECIMENTOS}

À FAPESP pela concessão da bolsa de Iniciação Científica (processo 2005/04131-6), à Juliana Perina Gândara e Rafael Muniz Pacchiega pela revisão deste texto. 


\begin{abstract}
Purpose: to quantitatively analyze the relationship between the spontaneous use of nouns and verbs by preschool children with normal language development, as well as to analyze the classification of the verbs used on the same speech sample. Methods: speech samples of sixty preschoolers were gathered in educational context through playful interaction. For data analysis the children were divided into three groups, paired by gender and age: GI (between 2:0 and 2:11 years), Gll (between 3:0 and 3:11 years) and GIII (between 4:0 and 4:11 years). Results: data analysis showed that the use of verbs prevailed in all age groups, with no difference between genders. Regarding types of verbs, both genders had similar performance: intransitive verbs were more frequent, followed by copula in $\mathrm{Gl}$ and GII and direct transitive verbs in GIII. Conclusion: the results showed that the preschoolers studied used more verbs than nouns since their second year of life. Intransitive verbs were the most frequently observed in all age ranges, followed by copula for two- and three-year-olds and by direct transitive verbs for four-year-olds. Finally, no significant differences were found between genders for any of the aspects studied.
\end{abstract}

KEYWORDS: Language Development; Child Language; Vocabulary; Child

\section{REFERÊNCIAS}

1. Navas ALGP. Neurodesenvolvimento e linguagem. In: Berlim C, Muszkat M, Miranda MC, organizadores. Neuropsicologia de desenvolvimento. São Paulo: Memnon; 2006. p. 93-105.

2. Vigotski LS. Pensamento e linguagem. São Paulo: Martins Fontes; 2005. 160 p.

3. Chen P, Tonietto L, Parente MA, Duvignau K, Gaume B. Does number of action labels predict an early acquisition of the conventional meaning of verbs? No prelo.

4. Borges LC, Salomão NMR. Aquisição da linguagem: considerações da perspectiva da interação social. Psicol Reflex Crit. 2003; 16:327-36.

5. Snow CE. Questões no estudo do input: sintonia, universalidade, diferenças individuais e evolutivas, e causas necessárias. In: Fletcher $\mathrm{P}$, Mcwhinney $B$, organizadores. Compêndio da linguagem da criança. Porto Alegre: Artes Médicas; 1997. p. 153-63.

6. Lidz J, Waxman S, Freedman J. What infants know about syntax but couldn't have learned: experimental evidence for syntactic structure at 18 months. Cognition. 2003; 89(3):B65-73.

7. Tomasello M. A usage-based approach to child language acquisition. Proceedings of The Berkeley Linguistics Society. 2000.

8. Tomasello M. The item-based nature of chuldren's early syntactic development. Trends Cogn Sci. 2000; 4(4):156-63.

9. Amorim A. Fonoaudiologia geral. Rio de Janeiro: Enelivros; $1982.116 \mathrm{p}$.

10. Rosa MC. Introdução à morfologia. São Paulo: Contexto; 2003. 156 p.
11. Marshall J. Noun-verb dissociations: evidence from acquisition and developmental and acquired impairments. J Neurolinguistics. 2003; 16:67-84.

12. Tomasello $M$, Kruger AC. Joint attention on actions: acquiring verbs in ostensive and non-ostensive contexts. J Child Lang. 1992; 19(2):311-33.

13. Naigles LR, Hoff-Ginsberg E. Why are some verbs learned before other verbs? effects of input frequency and structure on children's early verb use. J Child Lang. 1998; 25(1):95-120.

14. Goldfield BA. Nouns before verbs in comprehension vs. production: the view from pragmatics. J Child Lang. 2000; 27(3):501-20.

15. Skipp A, Windfuhr KL, Conti-Ramsden G. Children's grammatical categories of verb and noun: a comparative look at children with specific language impairment (SLI) and normal language (NL). Int $\mathrm{J}$ Lang Commun Dis. 2002; 37(3):253-71.

16. Barrett M. Desenvolvimento lexical inicial. In: Fletcher $P$, Mcwhinney B, organizadores. Compêndio da linguagem da criança. Porto Alegre: Artes Médicas; 1997. p. 299-321.

17. Geren J, Snedeker J, Ax L. Starting over: a preliminary study of early lexical and syntactic development in internationally adopted preschoolers. Semin Speech Lang. 2005; 26(1):44-53.

18. Sinigaglia J. Emergência dos verbos no português do Brasil. Relatório de Iniciação Científica - CNPQ - PIBIC; Universidade Federal de Santa Catarina; 2006.

19. Ambalu D, Chiat S, Pring T. When is it best to hear a verb? the effects of the timing and focus of verb models on children's learning of verbs. $J$ Child Lang. 1997; 24(1):25-34. 
20. Mobayyen F, Almeida RG. The influence of semantic and morphological complexity of verbs on sentence recall: implications for the nature of conceptual representation and category-specific deficits. Brain Cogn. 2005; 57(2):168-71.

21. Gillette J, Gleitman H, Gleitman L, Lederer A. Human simulations of vocabulary learning. Cognition. 1999; 73(2):135-76.

22. Forbes JN, Poulin-Dubois D. Representational change in young children's understanding of familiar verb meaning. J Child Lang. 1997; 24(2):389-406.

23. Naigles L. Children use syntax to learn verb meanings. J Child Lang. 1990; 17(2):357-74.

24. Naigles L. Young children's use of syntatic frames to derive meaning. In: Hirsh-Pasek K, Golinkoff RM. The origins of grammar: evidence from early language comprehension. Massachusetts: The MIT Press; 1999. p. 123-58.

25. Gleitman LR, Gillette J. O papel da sintaxe na aprendizagem dos verbos. In: Fletcher $P$, Mcwhinney $B$, organizadores. Compêndio da linguagem da criança. Porto Alegre: Artes Médicas; 1997. p. 341-52.

26. Weist RM, Pawlak A, Carapella J. Syntacticsemantic interface in the acquisition of verb morphology. J Child Lang. 2004; 31(1):31-60.
27. Kersten AW, Smith LB. Attention to novel objects during verb learning. Child Dev. 2002; 73(1):93-109.

28. Folli $R$, Harley $H$. What language says about the psychology of events. Trends Cogn Sci. 2006; 10(3):91-2.

29. Naigles LR, Lehrer N. Language-general and language-specific influences on children's acquisition of argument structure: a comparison of french and english. J Child Lang. 2002; 29(3):545-66.

30. Barbosa JB. O uso dos verbos no desenvolvimento da linguagem [periódico on line]. Revista Virtual de Estudos da Linguagem - Revel. 2005; 3(5). Disponível em: URL: http://www.revel.inf.br/ site2007/getfile.php?token $=11958555734747$ ed55 e32a\&id=Mzc=\&local=artigos.

31. Garcia AS. Verbos designativos do português [periódico on line]. Soletras - Revista do Departamento de Letras da UERJ. 2005. Disponível em: URL: http://www.filologia.org.br/soletras.

32. Lima CHR. Gramática normativa da língua portuguesa. Rio de Janeiro: José Olympio; 1982. 553 p. 33. Fluharty NB. Fluharty preschool speech and language screening tests. Pro-ed; 1978.

34. Brown R. A first language the early stages. Cambridge: Harvard University Press; 1973.
RECEBIDO EM: 15/05/2007

ACEITO EM: 16/10/2007

Endereço para correspondência:

Rua Cipotânea, 51

São Paulo - SP

CEP: 05360-160

Tel: (11) 30917453

E-mail: dmblopes@usp.br 